\title{
"MS4 Report": a Student-Led and Remote Model for Case-Based Learning in Preparation for Residency
}

\author{
Matthew $\operatorname{Tsai}^{1}$ • Dylan Koundakjian ${ }^{1} \cdot$ Emily Greenberger ${ }^{1,2,3}$ (i)
}

Accepted: 12 October 2020 / Published online: 27 October 2020

(C) International Association of Medical Science Educators 2020

\begin{abstract}
Case-based conference preparation is a valuable skill rarely covered in medical school training. We implemented an innovative program to teach fourth-year medical students to prepare and facilitate a virtual case presentation conference with faculty mentorship. Feedback survey results indicated improved confidence in case presentation and in establishing a broad differential.
\end{abstract}

Keywords Clinical teaching $\cdot$ Case-based learning $\cdot$ Peer education $\cdot$ Remote learning $\cdot$ Medical education

The arrival of COVID-19 within the USA prompted unprecedented changes in medical education, including the American Association of Medical Colleges' guidance supporting the pausing of medical student clinical rotations [1]. Medical students at the Larner College of Medicine at the University of Vermont (LCOM) required new outlets to practice advanced clinical reasoning skills like case-based presentations, formulating differential diagnoses, assessments, and plans outside of inpatient and outpatient clinical settings. Our students also lacked experience in preparing case-based learning presentations, a learning modality adopted by many residency programs and perceived by residents as more effective when delivered by a peer educator [2]. While students are frequent attendees at resident conferences such as internal medicine "Morning Report," there are few known initiatives which teach students to design and present case conferences [3].

We developed an innovative program called "MS4 Report," in which fourth-year medical students were asked to virtually present real clinical cases of their choosing to a group of their peers and an invited faculty member and to guide the clinical discussion and learning. Unlike other case presentations, which are usually led by medical residents, this program was led by

Emily Greenberger

Emily.Greenberger@uvmhealth.org

1 Robert D Larner MD College of Medicine at the University of Vermont, Burlington, VT, USA

2 Department of Medicine, University of Vermont Medical Center, Burlington, VT, USA

3 UVM Medical Center Adult Primary Care, 1 Timber Lane, South Burlington, VT 05403, USA and for fourth-year medical students, multidisciplinary in breadth, and limited to a single presenter per case [3]. Following the Kern Model for evaluating an educational program, we sought to address several learning objectives, which we assessed by surveys of student attitudes [4]. These objectives included the following: (i) Participants will gain comfort with forming a broad differential diagnosis for a given clinical presentation; (ii) Participants will feel more engaged in their medical education; (iii) Participants will gain comfort with contributing to a case discussion; (iv) Presenters will gain comfort with preparing a case presentation; (v) Presenters will gain confidence leading a case-based discussion; (vi) Presenters will gain confidence communicating learning objectives.

To implement, we recruited one General Internal Medicine faculty member to mentor each student in presentation design to ensure effectiveness of delivery and teaching points. Two medical student leaders coordinated student sign-ups for each week's conference and provided technical and logistical assistance including scheduling presentations and recruiting faculty discussants. Faculty discussants helped to support student presenters by providing expert input and clinical pearls throughout the presentation. Seven fourth-year students volunteered to each present a one-hour case presentation to a group of fourth-year student attendees. Any fourth-year student could attend. Seven presentations were scheduled on a weekly basis from late April to May 2020. Presenters used Microsoft PowerPoint and delivered each conference on the virtual platform Zoom. Topics varied by each case and included emergency medicine decision-making, neonatal resuscitation, anemia workup, liver disease sequelae, and a morbidity and mortality discussion. Presenters reviewed their presentations with the faculty administrator beforehand to identify learning objectives. 
Table 1 MS4 report quality survey results $\left(n=9\right.$ attendees ${ }^{\mathrm{a}}$, 4 presenters)
Quality measure

Average agreement on a standard 1-5 Likert scale, 5 indicating strongly agree (standard deviation)

\begin{tabular}{ll}
\hline Increased comfort with forming a broad differential & $4.2(0.44)$ \\
Feel more engagement in medical education & $4.4(0.53)$ \\
Increased comfort contributing to a case discussion & $4.3(0.71)$ \\
Preference for the student-led format of MS4 Report over & $3.8(0.83)$ \\
$\quad$ the resident-led format of other conferences & $4.7(0.5)$ \\
$\quad$ Interest in participating in a similar activity in student's & $4.3(0.71)$ \\
$\quad$ Entended specialty of practice & $4.6(0.53)$ \\
ZOOM variety among MS4 Report cases & \\
Presenters only & \\
Increased confidence in ability to prepare a case presentation & $4.5(0.58)$ \\
Increased confidence in ability to lead a case-based discussion & $4.5(0.58)$ \\
Increased comfort with clinical content presented & $4.0(0.82)$ \\
\hline
\end{tabular}

Student administrators excluded from the number of attendees and the number of presenters reported above.

${ }^{\text {a }}$ Average number of MS4 reports attended 4.1 (2.3)
Excluding student presenters and leaders, session attendance ranged from two to eleven attendees (mean of 6.57 attendees). On the day of the last and seventh session, all attendees and presenters were invited to provide feedback via survey. We obtained IRB exemption prior to survey development. Results are listed in Table 1. Survey participants were asked to rate how strongly they agreed or disagreed with several quality measures related to their participation in MS4 Report on a standard 1-5 Likert scale (5 indicating strongly agree). Notably, presenters most strongly agreed that they experienced increased confidence in their ability to prepare a case presentation (mean 4.5, SD 0.58) and to lead a casebased discussion (mean 4.5, SD 0.58). Attendees collectively indicated increased comfort with forming a broad differential diagnosis, assessment, and plan, increased engagement in their medical education, and increased comfort with contributing to a case discussion. Interestingly, agreement was not as strong in attendee preference for a student-led format like MS4 Report compared to a resident-led format, a finding contradictory to a separate study of third-year student group presentations [3]. One explanation is that the virtual platform, though populated by peers, was still perceived as impersonal. Limitations of our report include its small sample size, short duration of study, and selection bias, considering participation was voluntary.

The MS4 Report model may improve students' abilities to prepare, deliver, and facilitate a case presentation conference with faculty mentorship in preparation for residency. Attendees may also benefit from improved engagement in their training and increased confidence in their clinical reasoning skills. Additional research may clarify the role similar programs could play in a post-COVID medical education curriculum.

Acknowledgments The authors gratefully acknowledge the guidance of The Larner College of Medicine Teaching Academy and Dr. Halle Sobel.

Data Availability Data is included in Table 1.

\section{Compliance with Ethical Standards}

Conflict of Interest The authors declare that they have no conflict of interest.

Code Availability Not applicable.

\section{References}

1. Important Guidance for Medical Students on Clinical Rotations During the Coronavirus (COVID-19) Outbreak. AAMC Press Releases. 2020. Web. Accessed 28 May 2020. https://www.aamc. org/news-insights/press-releases/important-guidance-medicalstudents-clinical-rotations-during-coronavirus-covid-19-outbreak

2. Fraser T, Sargsyan Z, Baggett TP, Baggett M. quantitative study of the characteristics of effective internal medicine noon conference presentations. J Grad Med Educ. 2016;8(2):185-90. https://doi.org/ 10.4300/JGME-D-15-00132.1.

3. Jafri NF, Nadgir R, Slanetz PJ. Student-facilitated radiology-pathology correlation conferences: an experiential educational tool to teach multidisciplinary patient care. J Am Coll Radiol. 2010;7(7):512-6. https://doi.org/10.1016/j.jacr.2010.02.010.

4. Kern DE, Thomas PA, Hughes MT. Curriculum Development for Medical Education: A Six-Step Approach. 2nd ed. Baltimore: The John's Hopkins University Press; 2009.

Publisher's Note Springer Nature remains neutral with regard to jurisdictional claims in published maps and institutional affiliations. 